\title{
Isolation of chloroplast FtsZ and AtpC, and analysis of protein targeting into the complex chloroplast of the haptophyte Pavlova pinguis
}

\author{
Toshikazu Nishikawa, Hiroyuki Kajitani, Mayuko Sato, Yuko Mogi, \\ Yohsuke Moriyama and Shigeyuki Kawano* \\ Department of Integrated Biosciences, Graduate School of Frontier Sciences, University of \\ Tokyo, Bldg. FSB-601, 5-1-5 Kashiwanoha, Kashiwa, Chiba 277-8562, Japan
}

Received April 23, 2010; accepted June 23, 2010

\begin{abstract}
Summary Haptophytes are abundant phytoplankton that possess 4 membrane-bound chloroplasts. These chloroplasts originated from a red alga that was taken up by a eukaryotic host cell. No previous work has reported on the protein targeting of haptophytes, except for computational analyses. We isolated the two genes encoding chloroplast proteins AtpC1 and FtsZ from Pavlova pinguis and analyzed their molecular structure. These proteins had a bipartite sequence at the Nterminus, which consisted of an endoplasmic reticulum (ER) signal sequence followed by a chloroplast transit peptide. To demonstrate the functionality of the ER signal sequences and the chloroplast targeting sequences in vivo, we fused the predicted ER signal sequence, chloroplast transit peptide, and bipartite sequence of $\mathrm{AtpC} 1$ and $\mathrm{FtsZ}$ individually to the $\mathrm{N}$-terminus of green fluorescent protein (sGFP). This was then introduced into cultured tobacco cells. Microscopic observation revealed that the predicted ER signal sequences of AtpC1 and FtsZ had the ability to localize to the ER, and the following amino acids worked as a chloroplast transit peptide in the tobacco cells. We concluded that the AtpC1 and FtsZ of P. pinguis have a bipartite sequence at the $\mathrm{N}$-terminus and a molecular structure in common with other independently established secondarily endosymbiotic algae.
\end{abstract}

Keywords: bipartite targeting sequence, haptophyte, FtsZ

It is now widely accepted that the chloroplast arose by primary endosymbiosis (Delwiche and Palmer 1997, Martin and Herrmann 1998, Martin and Müller 1998). A formerly free-living cyanobacterium became a chloroplast, and the genes for most chloroplast proteins were transferred from the chloroplast genome to the nucleus and subsequently had to be equipped with chloroplasttargeting sequences. The primary chloroplast is bound by two membranes and was thought to have originated in a single endosymbiotic event in their common ancestor (McFadden 2001, RodríguezEzpeleta et al. 2005).

A variety of algal genera like diatoms, brown algae, cryptophytes, and haptophytes had experienced a second endosymbiosis step involving a red alga taken up by a eukaryotic host cell (Hempel et al. 2007). In these groups of algae, chloroplasts have additional membrane(s). Most genes encoding chloroplast proteins have been transferred a second time (from the nucleus of the endosymbiont to the nucleus of the secondary host) and must have been equipped with additional targeting signals to cross more than two chloroplast membranes (Kilian and Kroth 2004). The nucleus-encoded chloroplast proteins in these organisms are thought to have bipartite presequences consisting of a signal sequence followed by a transit peptide (Lang et al. 1998, Sulli et al. 1999, Waller et al. 2000).

To gain new insight into the import of proteins into the haptophyte chloroplast, we isolated two

\footnotetext{
*Corresponding author, e-mail: kawano@k.u-tokyo.ac.jp
} 
genes coding chloroplast proteins AtpC1 and FtsZ from P. pinguis. This is the first report of chloroplast-targeting sequence analyses in a haptophyte.

Materials and Methods

\section{Cell culture}

Pavlova pinguis cells were cultured in $\mathrm{f} / 2$ medium (Guillard 1972). The cells were grown under continuous light $\left(50 \mu \mathrm{E} \mathrm{m}^{-2} \mathrm{~s}^{-1}\right)$ at $23^{\circ} \mathrm{C}$ without shaking or bubbling.

\section{Transmission electron microscopy}

Cells were frozen in a high-pressure freezing apparatus (HPM-010; Bal-Tec AG, Liechtenstein). The frozen cells were transferred to $2 \% \mathrm{OsO}_{4}$ in dry acetone at $-80^{\circ} \mathrm{C}$ and incubated at $-80^{\circ} \mathrm{C}$ for $108 \mathrm{~h}$. Subsequently, the samples were warmed gradually from $-80^{\circ} \mathrm{C}$ to $0^{\circ} \mathrm{C}$ over $11 \mathrm{~h}$, held for $1.5 \mathrm{~h}$ at $0^{\circ} \mathrm{C}$, warmed again from $0^{\circ} \mathrm{C}$ to $23^{\circ} \mathrm{C}$ over $4 \mathrm{~h}$, and then incubated for $2 \mathrm{~h}$ at $24^{\circ} \mathrm{C}$ (Leica EM AFS; Leica Microsystems, Germany). The samples were washed with dry acetone three times at room temperature and infiltrated with increasing concentrations of Spurr's resin in dry acetone, and finally with $100 \%$ Spurr's resin. Ultrathin sections were stained with 3\% uranyl acetate for $2 \mathrm{~h}$ and with lead citrate for $10 \mathrm{~min}$ and examined under an electron microscope (H-7600; Hitachi High-Tech, Japan) at $100 \mathrm{kV}$.

\section{Genomic DNA preparation and PCR with degenerate primers}

Total DNA of $P$. pinguis was extracted with an Easy-DNA Kit (Invitrogen, USA). To obtain fragments of the FtsZ and AtpCl genes from P. pinguis, we conducted nested polymerase chain reaction (PCR) surveys using degenerate primers based on the amino acid (aa) sequences of these genes. Partial sequences of AtpCl were obtained using the primer pair f1 5'-AARAACACCCRRAAGATCAC-3' and r1 5'-GGRTCCTGYTCGAAGATCAT-3' in the first PCR reaction, and f2 5'-GTBCGWCGYGCWCARGAYGC-3' and r2 5'-GARTTGTABCYKCCRCARAG-3' in the second. The degenerate primers for the FtsZ gene were 5'-GAYYTNGTNTTYGNACNGC-3' and 5'-GCNCCRAACATDATRYTBGC-3' in the first PCR reaction, and 5'-GGHATGGGHGGHGGHACNGG-3' and 5'-ACRTCNGCRAARTCHACRTT-3' in the second. PCR was performed for 30 cycles at $94^{\circ} \mathrm{C}$ for $1 \mathrm{~min}, 50^{\circ} \mathrm{C}$ for $1 \mathrm{~min}$, and $72^{\circ} \mathrm{C}$ for $30 \mathrm{~s}$, with a final $5 \mathrm{~min}$ at $72^{\circ} \mathrm{C}$ with ExTaq (Takara Bio, Japan).

The PCR fragments were cloned into a pGEM-T Easy Vector (Promega, USA) and then sequenced with an ABI PRISM 3100 Genetic Analyzer (Applied Biosystems, USA) using a BigDye Terminator Cycle Sequencing Ready Reaction Kit V2.0 (Applied Biosystems) following the manufacturer's instructions.

\section{Inverse PCR}

To obtain full-length sequences of the AtpCl and Fts Z genes, inverse PCR was performed. Using restriction enzymes with no recognition sites in the sequence of the degenerate PCR products, $4 \mu \mathrm{g}$ of total DNA was digested. The digested DNA was self-ligated with T4 DNA ligase (New England Biolabs, USA). After treatment with phenol/chloroform solution, the digested DNA was precipitated with ethanol and then used as PCR template. PCR was performed for 30 cycles at $94^{\circ} \mathrm{C}$ for $1 \mathrm{~min}, 65^{\circ} \mathrm{C}$ for $1 \mathrm{~min}$, and $72^{\circ} \mathrm{C}$ for $2 \mathrm{~min}$, with a final $5 \mathrm{~min}$ at $72^{\circ} \mathrm{C}$ with ExTaq using specific primer sets. The PCR products were purified as described above and cloned into a pGEM-T Easy Vector. Sequencing was carried out as described above, and primer walking was performed at least twice. 
Transformation of cultured tobacco cells

The predicted targeting sequences of AtpC1 and FtsZ from P. pinguis were amplified by PCR. The following primer sets were used: for analyzing AtpC1 sequences, atpc_ER_N 5' GGGGTCGACATGTTCCGCTCAT-3' and atpc_ER_C 5'-GGCCATGGCGGCCGAGGCCGAC-3' for the ER signal sequence, atpc_CP_N 5'-GGGGTCGACATGTTCAACATGG-3' and atpc_CP_C 5'-GGCCATGGCCGCGTTGCCCTGC-3' for the chloroplast transit peptide, and atpc_ER_N and atpc_CP_C for the bipartite targeting sequence; for FtsZ, ftsZ_ER_N 5'-GGGTCGACATGCGGCGCACGA-3' and ftsZ_ER_C 5'-GGCCATGGCGGCGGTGGCTGCT-3' for the ER signal sequence, ftsZ_CP_N 5'-GGGGTCGACATGTATAGCAGTC-3' and ftsZ_CP_C 5'-GGCCATGGCCAGAGGGAGAAG-3' for the chloroplast transit peptide, and ftsZ_ER_N and ftsZ_CP_C for the bipartite targeting sequence.

The PCR products were digested with SalI and NcoI, and cloned into the corresponding site of pUC18 containing a green fluorescent protein (GFP) gene [sGFP(S65T)] under the control of a strong double cauliflower mosaic virus (CaMV) 35S promoter (Niwa et al. 1999). The resulting constructs (35S::targeting sequence: sGFP) were introduced into protoplasts of Nicotiana tabacum S401 L cv. Samsun cultured cells using polyethylene glycol. Tobacco suspension-cultured cells were maintained in modified MS medium at $20^{\circ} \mathrm{C}$ under illumination. The modified method of Forreiter et al. (1997) was used to introduce DNA into cells. Transformed protoplasts were incubated with gentle agitation at $23^{\circ} \mathrm{C}$ for $12 \mathrm{~h}$ in the dark.

\section{Results}

Morphology of $\mathrm{P}$. pinguis cells

Transmission electron microscopic observation of $P$. pinguis showed one nucleus, some scattered mitochondria, a distinct Golgi apparatus, and large chloroplasts (Fig. 1). The secondary acquired chloroplast has four membranes; the inner two are derived from the original chloroplast, and outer two are of host-cell origin. The outermost membrane is contiguous with the rough endoplasmic reticulum (ER) and the outer nuclear envelope (Cavalier-Smith 2003, Keeling 2009). The precise structure of these four membranes is unclear due to the highpressure freezing method, but surrounding ribosomes are obvious on the outermost membrane (Fig. 1).

Isolation of haptophyte genes encoding chloroplast proteins

To isolate the full-length genes of chloroplast proteins AtpC1 and FtsZ from $P$. pinguis, partial sequences were amplified from genomic DNA using a degenerate PCR method. Subsequently, inverse PCR was performed with specific primers based on the partial sequences. As a result, we obtained one full-length AtpC1 (PpAtpC1; $1014 \mathrm{bp)} \mathrm{gene}$ and one full-length FtsZ gene (PpFtsZ; 1296 $\mathrm{bp})$. These two sequences have been deposited in DDBJ, under the following accession numbers: PpAtpCl (AB557976) and PpFtsZ

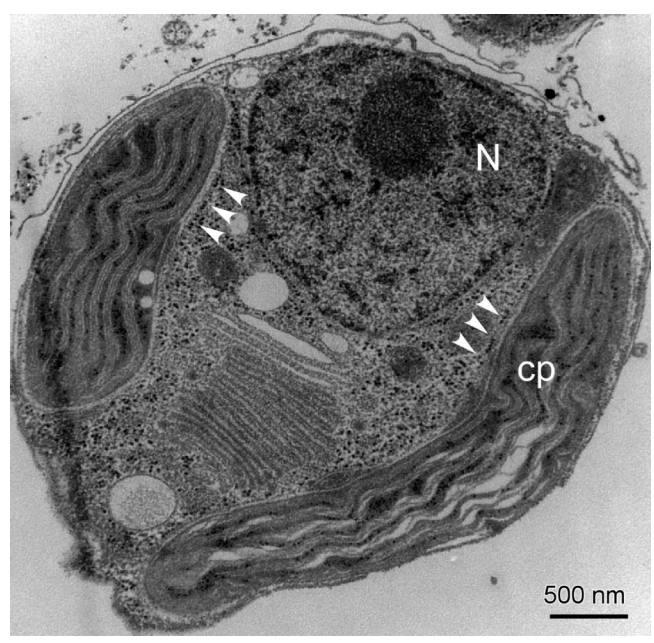

Fig. 1. Transmission electron microscopic image of Pavlova pinguis. The chloroplasts are surrounded by the epiplastid membrane, which is studded with ribosomes and connected to the rough ER and outer nuclear envelope. N, nuclei; $\mathrm{cp}$, chloroplast; arrowheads, ribosomes on the epiplastid membrane. Scale bar $=500 \mathrm{~nm}$. 

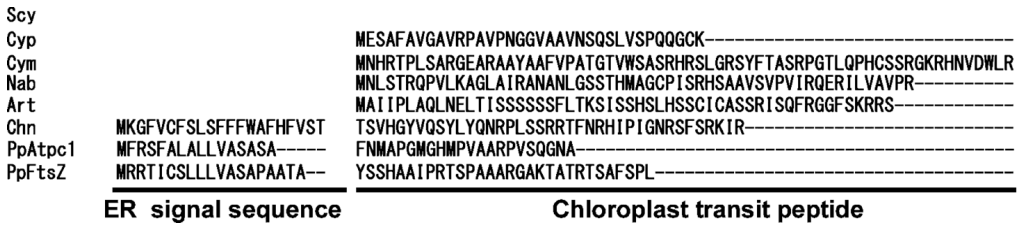

MTDPMPINNSYGFNRDGSLSGFDAL AEARAQFSYSKKAANSAKLQATRRG SAMRVQANGASYPGRGNDRQLSSLP ASYSNANYGPMGGDARIKVVGVGGG DSTRSKSMRLRCSFSPMESAR IKVI DPSGVSVKLGFHHHLRGSTSSSGGG MFRSFALALLVASASAFNMAPGMGH Chloroplast transit peptide

Mature protein

Fig. 2. The N-termini of PpAtpC1 and PpFtsZ were compared to the FtsZs of the cyanobacterium Synechocystis sp. PCC6803 (Scy), glaucophyte Cyanophora paradoxa (Cyp), red alga Cyanidioschyzon merolae (Cym), green alga Nannochloris bacillaris (Nab), land plant Arabidopsis thaliana (Art), and diatom Chaetoceros neogracile $(\mathrm{Chn})$. The organelle targeting sequences of $C$. neogracile, $\mathrm{PpAtpC1}$ and $\mathrm{PpFtsZ}$, were predicted using SignalP and ChloroP, and aligned with the other FtsZ proteins. The ER signal sequence, chloroplast transit peptide, and mature proteins are underlined. The cyanobacterium has no targeting sequence, while the primary endosymbiotic algae (glaucophyte, red alga, and green alga) have a chloroplast transit peptide at the N-terminus of FtsZ. Conversely, the secondary symbiotic algae (diatom and haptophyte Pavlova pinguis) are predicted to have an additional ER signal sequence at the N-terminus of each protein.

(AB557975). According to the inverse PCR, PpAtpC1 has no intron, while the PpFtsZ gene coding regions are interrupted by three introns.

\section{Prediction of the N-terminus organelle transit peptide}

Computational analysis of the derived sequences using the programs SignalP (http:// www.cbs.dtu.dk/services/SignalP/; Nielsen et al. 1999) and ChloroP (http://www.cbs.dtu.dk/ services/ChloroP/; Emanuelsson et al. 1999) predicted that the protein sequences of PpAtpC1 and PpFtsZ had N-terminal bipartite presequences, which consist of an ER presequence followed by a chloroplast presequence. The N-termini of PpAtpC1 and PpFtsZ were aligned with known FtsZ orthologs of other species (Fig. 2). PpAtpC1 contains a 16-aa ER signal sequence and a 22-aa chloroplast transit peptide at the N-terminus. PpFtsZ has a 19-aa ER signal sequence followed by a 30-aa chloroplast transit peptide.

\section{Transient assay of the fusion protein of SGFP to the organelle signal sequence}

To demonstrate the functionality of the ER signal sequence and chloroplast targeting sequences in vivo, we genetically fused the predicted ER signal sequence, chloroplast transit peptide, and bipartite sequence of AtpC1 and FtsZ individually to the N-terminus of sGFP, and used a tobacco culture cell transformation system to visualize the ability of the predicted transit peptides using a plant expression vector (Figs. 3 and 4). When the fusion protein of the ER signal sequence:sGFP, for which the N-terminus sequence was derived from PpAtpC1 or PpFtsZ, was expressed in tobacco cells, fluorescence accumulated around the cytosol as a netlike structure (Figs. $3 \mathrm{a}-\mathrm{d}$ and $4 \mathrm{a}-\mathrm{d}$, respectively). This localization of the accumulated ER signal sequence:sGFP fluorescence was similar to the ER-Tracker stained structures (data not shown). The predicted chloroplast transit peptides of PpAtpc1 or PpFtsZ were also fused to sGFP and expressed in cultured tobacco cells (Figs. $3 \mathrm{e}-\mathrm{h}$ and $4 \mathrm{e}-\mathrm{h}$, respectively). In both cases, the fluorescence of the sGFP clearly co-localized to the chloroplast autofluorescence. Therefore, these putative chloroplast transit peptides successfully delivered the GFP into the chloroplast, even in tobacco cells. To confirm the dominance of the ER signal sequence and chloroplast transit peptide, the bipartite sequence of PpAtpC1 or PpFtsZ was fused to sGFP and expressed in tobacco cells (Figs. 3i-1 and $4 \mathrm{i}-1$, respectively). Microscopic observations showed that the localization of the bipartite sequence:sGFP fluorescence was similar to that of the ER signal sequence:sGFP, irrespective of the origin of the bipartite sequence. 

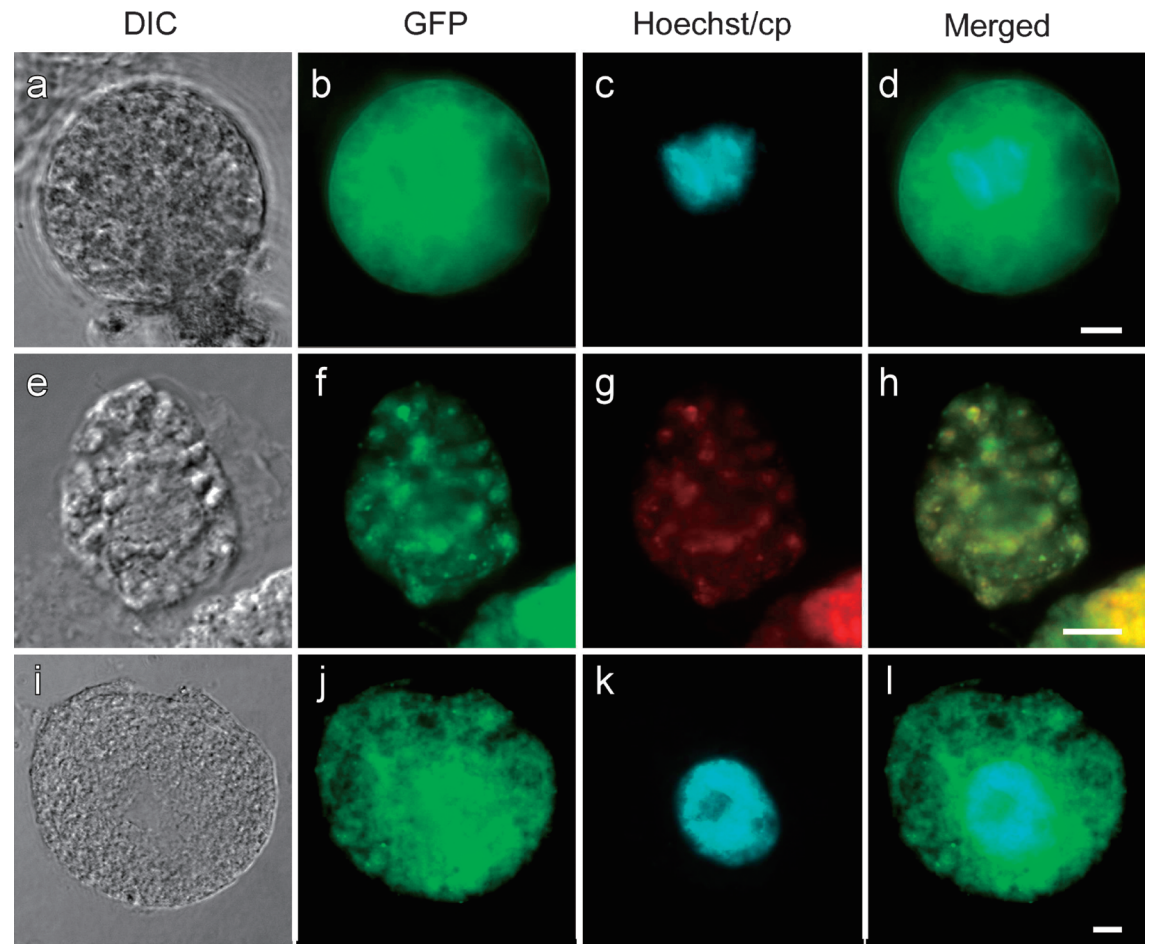

Fig. 3. Transient expression of the GFP-tagged organelle targeting sequences from PpAtpC1 in cultured tobacco cells. The transformed cells were stained with Hoechst 33342 and observed under differential interference contrast (DIC) and fluorescence microscopy. (a-d) ER signal sequence: sGFP, (e-h) chloroplast transit peptide: sGFP, (i-1) bipartite sequence: sGFP. c and k, nuclei; g, chlorophyll autofluorescence. The fusion protein of the ER signal sequence: sGFP and bipartite sequence: sGFP localized around the cell nuclei, but the chloroplast transit peptide: sGFP clearly accumulated within the chloroplast Scale bar $=10 \mu \mathrm{m}$.

\section{Discussion}

Almost all known haptophytes are photosynthetic and possess four membrane-bound chloroplasts whereby the outermost membrane is continuous with the host rough ER (CavalierSmith 2003, Keeling 2009). The chloroplast of haptophyte was acquired by secondary endosymbiosis, and the chloroplast proteins must have subsequently been equipped with unique targeting signals for crossing the additional chloroplast membranes.

We isolated one AtpCl and one FtsZ gene from the haptophyte P. pinguis. AtpC1 is a protein related to photosynthesis, and FtsZ is a key protein in the constriction and binary fission of the chloroplasts and bacterial cells. Chloroplast $F t s Z$ genes are found in many algal species, including the unicellular glaucophyte Cyanophora paradoxa (Sato et al. 2005), the red alga Cyanidium caldarium (Takahara et al. 2000), and the green alga Nannochloris bacillaris (Koide et al. 2004). Since two or more FtsZ paralogs exist in red algae, green algae, and even in the secondary endosymbiotic algae Thalassiosira pseudonana and Phaeodactylum tricornutum (Armbrust et al. 2004, Bowler et al. 2008), searching for other FtsZ paralogs in P. pinguis is necessary.

Compared to the AtpC1 and FtsZ of other species, the entire aa sequences and functional domains are highly conserved. PpFtsZ conserved the C-terminal domain (Löwe and Amos 1998) and $\mathrm{C}$-terminal core domain $(\mathrm{D} / \mathrm{E}-\mathrm{I} / \mathrm{V}-\mathrm{P}-\mathrm{X}-\mathrm{F} / \mathrm{Y}-\mathrm{L}$, where $\mathrm{P}$ is invariable) in cyanobacterial FtsZ, the chloroplast FtsZ2 of green-lineage plants and glaucophytes, and the chloroplast FtsZA of red algal lineages (red algae and stramenopiles) (Miyagishima et al. 2004); in addition, PpFtsZ is 

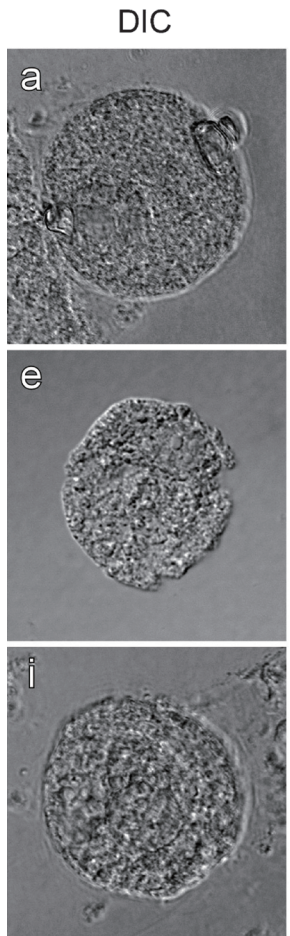

Hoechst/cp

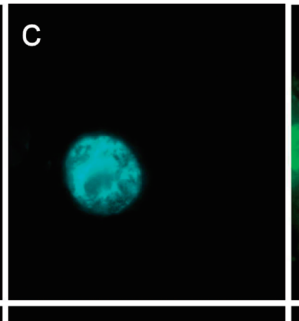

g
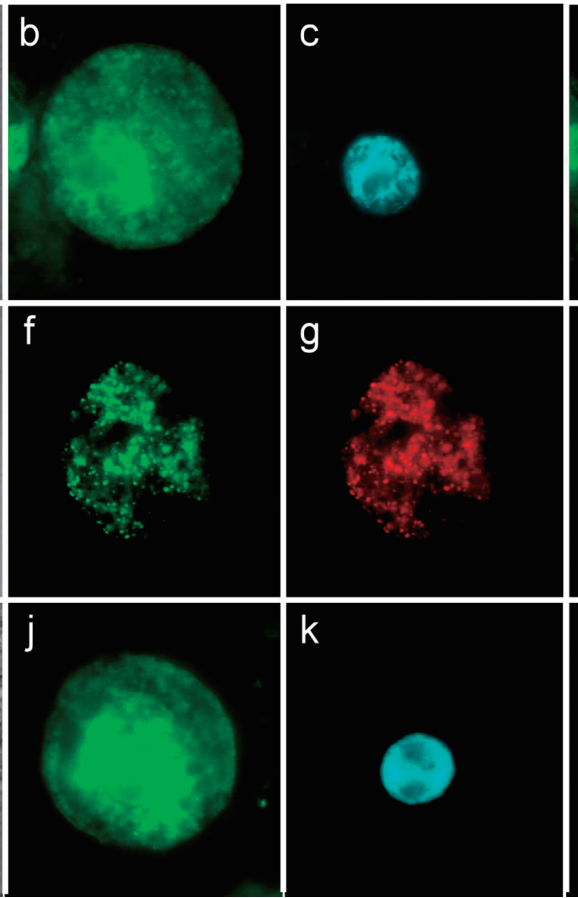

\section{Merged}

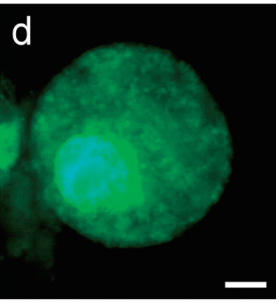

$\mathrm{h}$

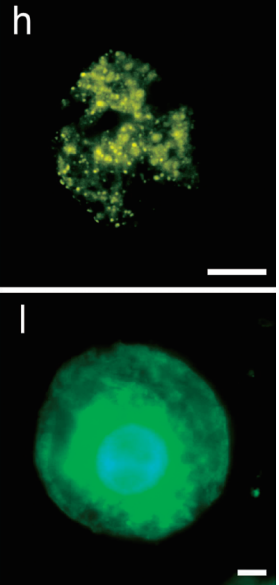

Fig. 4. Transient expression of the GFP-tagged organelle targeting sequences from PpFtsZ in cultured tobacco cells. Organelle targeting sequences from PpFtsZ were fused to sGFP and expressed in the tobacco cells. The transformed cells were stained with Hoechst 33342, and observed under DIC and fluorescence microscopy. (a-d) ER signal sequence: sGFP, (e-h) chloroplast transit peptide: sGFP, (i-1) bipartite sequence: sGFP. c and k, nuclei; g, chlorophyll autofluorescence. The localization patterns of the fusion proteins are almost the same as those for AtpC1. Scale bar $=10 \mu \mathrm{m}$.

believed to be involved in protein-protein interactions (Osteryoung and McAndrew 2001, Stokes and Osteryoung 2003), with some alterations (S-M-P-G-F-L).

The bipartite transit peptides are necessary for targeting proteins from the cytosol to the chloroplast of secondary endosymbiotic organisms; however, no previous study had reported on protein targeting of haptophytes, except for a computational analysis (Patron et al. 2007). We identified the predicted N-terminal bipartite transit peptides of PpAtpC1 and PpFtsZ (Fig. 2). Their signal sequence cleavage sites are ASAF and ATAY, respectively, whereas the highly conserved signal sequence cleavage sites in diatoms are A-S/F-A-F/P (Kroth 2002, Kilian and Kroth 2005, Gould et al. 2006, Patron et al., 2007). The predicted ER signal sequences of AtpC1 and FtsZ from the haptophyte $P$. pinguis have the ability to localize into ER, and the subsequent amino acids work as a chloroplast transit peptide in tobacco cells (Figs. 3 and 4). The entire N-terminal bipartite sequence of PpAtpC1 and PpFtsZ has no ability to localize into tobacco chloroplasts because its chloroplast is not surrounded by an epiplastid membrane, which specifically exists in the secondary acquired chloroplast and is continuous with the ER.

We conclude that the chloroplast protein of haptophytes has a molecular structure in common with other independently established secondary endosymbiotic algae.

\section{Acknowledgment}

This research was supported in part by the River Fund in charge of the Foundation of River 
and Watershed Environment Management (FOREM), Japan, and by a Grant-in-Aid for Scientific Research (B) (20370025) from the Ministry of Education, Culture, Sports, Science and Technology (MEXT), Japan.

\section{References}

Armbrust, E. V., Berges, J. A., Bowler, C., et al. 2004. The genome of the diatom Thalassiosira pseudonana: ecology, evolution, and metabolism. Science 306: 79-86.

Bowler, C., Allen, A. E., Badger, J. H., et al. 2008. The Phaeodactylum genome reveals the evolutionary history of diatom genomes. Nature 456: 239-244.

Cavalier-Smith, T. 2003. Genomic reduction and evolution of novel genetic membranes and protein-targeting machinery in eukaryote-eukaryote chimaeras (meta-algae). Philos. Trans. R. Soc. Lond. B. Biol. Sci. 358: 109-134.

Delwiche, C. F. and Palmer, J. D. 1997. The origin of plastids and their spread via secondary endosymbiosis. Origins of algae and their plastids. Springer-Verlag, Wien, New York. 53-86.

Emanuelsson, O., Nielsen, H. and von Heijne, G. ChloroP. 1999. ChloroP, a neural network-based method for predicting chloroplast transit peptides and their cleavage sites. Protein Science 8: 978-984.

Forreiter, C., Kirschner, M. and Nover, L. 1997. Stable transformation of an Arabidopsis cell suspension culture with firefly luciferase providing a cellular system for analysis of chaperone activity in vivo. Plant Cell 9: 2171-2181.

Gould, S. B., Sommer, M. S., Kroth, P. G., Gile, G. H., Keeling, P. J. and Maier, U. G. 2006. Nucleus-to-nucleus gene transfer and protein retargeting into a remnant cytoplasm of cryptophytes and diatoms. Mol. Biol. Evol. 23: 2413-2422.

Guillard, R. R. L. 1975. Culture of phytoplankton for feeding marine invertebrates. In "Culture of Marine Invertebrate Animals" (eds: Smith, W. L. and Chanley, M. H.) Plenum Press, New York, USA. 29-60.

Hempel, F., Bozarth, A., Sommer, M. S., Zauner, S., Przyborski, J. M. and Maier, U. G. 2007. Transport of nuclear-encoded proteins into secondarily evolved plastids. Biol. Chem. 388: 899-906.

Keeling, P. J. 2009. Chromalveolates and the evolution of plastids by secondary endosymbiosis. J. Eukaryot. Microbiol. 56: $1-8$.

Kilian, O. and Kroth, P. G. 2004. Presequence acquisition during secondary endocytobiosis and the possible role of introns. J. Mol. Evol. 58: 712-721.

Koide, T., Yamazaki, T., Yamamoto, M., et al. 2004. Molecular divergence and characterization of two chloroplast division genes, FtsZ1 and FtsZ2, in the unicellular green alga Nannochloris bacillaris (Chlorophyta). J. Phycol. 40: $546-556$.

Kroth, P. G. 2002. Protein transport into secondary plastids and the evolution of primary and secondary plastids. Int. Rev. Cytol. 221: 191-255.

Lang, M., Apt, K. E. and Kroth, P. G. 1998. Protein transport into "complex" diatom plastids utilizes two different targeting signals. J. Biol. Chem. 273: 30973-30978.

Löwe, J. and Amos, L. A. 1998. Crystal structure of the bacterial cell-division protein FtsZ. Nature 391: 203-206.

Martin, W. and Herrmann, R. H. 1998. Gene transfer from organelles to the nucleus: how much, what happens, and why? Plant Physiol. 118: 9-17.

— and Müller, M. 1998. The hydrogen hypothesis for the first eukaryote. Nature 392: 37-41.

McFadden, G. I. 2001 Primary and secondary endosymbiosis and the origin of plastids. J. Phycol. 37: 951-959.

Miyagishima, S. Y., Nozaki, H., Nishida, K., Nishida, K., Matsuzaki, M. and Kuroiwa T. 2004. Two types of FtsZ proteins in mitochondria and red-lineage chloroplasts: the duplication of FtsZ is implicated in endosymbiosis. J. Mol. Evol. 58: 291-303.

Nielsen, H., Engelbrecht, J., Brunak, S. and Heijne, G. 1999. Identification of prokaryotic and eukaryotic signal peptides and prediction of their cleavage sites. Protein Eng. 10: 1-6.

Niwa, Y., Hirano, T., Yoshimoto, K., Shimizu, M. and Kobayashi, H. 1999. Non-invasive quantitative detection and applications of non-toxic, S65T-type green fluorescent protein in living plants. Plant J. 18: 455-463.

Osteryoung, K. W. and McAndrew, R. S. 2001. The plastid division machine. Annu. Rev. Plant Physiol. Plant Mol. Biol. 52: 315-333.

Patron, N. J., Inagaki, Y. and Keeling, P. J. 2007. Multiple gene phylogenies support the monophyly of cryptomonad and haptophyte host lineages. Curr. Biol. 17: 887-991.

Rodríguez-Ezpeleta, N., Brinkmann, H., Burey, S. C. et al., 2005 Monophyly of primary photosynthetic eukaryotes: green plants, red algae, and glaucophytes. Curr. Biol. 15: 1325-1330.

Sato, M., Nishikawa, T., Yamazaki, T., and Kawano, S. 2005. Isolation of the plastid FtsZ gene from Cyanophora paradoxa (Glaucocystophyceae, Glaucocystophyta). Phycol. Res. 53: 93-96.

Stokes, K. D., and Osteryoung, K. W. 2003. Early divergence of the FtsZ1 and FtsZ2 plastid division gene families in 
photosynthetic eukaryotes. Gene 320: 97-108.

Sulli, C., Fang, Z. W., Muchhal, U. S., and Schwartzbach, S. D. 1999. Topology of Euglena chloroplast protein precursors within endoplasmic reticulum to Golgi to chloroplast transport vesicles. J. Biol. Chem. 274: 457-463.

Takahara, M., Takahashi, H., Matsunaga, S., Sakai, A., Kawano, S., and Kuroiwa, T. 2000. Isolation, characterization, and chromosomal mapping of an $f t s Z$ gene from the unicellular primitive red alga Cyanidium caldarium RK-1. Curr. Genet. 37: 143-151.

Waller, R. F., Reed, M. B., Cowman, A. F. and McFadden, G. I. 2000. Protein trafficking to the plastid of Plasmodium falciparum is via the secretory pathway. EMBO J. 19: 1794-1802. 